\title{
Hydra: A Powerful Biological Model ${ }^{*}$
}

\author{
Surendra Ghaskadbi
}

Hydra, a freshwater diploblast, with a simple but defined body plan, an organized nervous system, and the presence of stem cells, is one of the oldest model organisms used in biology. It exhibits many embryonic features even as an adult, a spectacular ability of regeneration, and lack of organismal aging. Hydra can provide insights into how complex animal forms evolved and is waiting to be better utilized in teaching.

To understand biological processes, scientists often choose and use a small number of species based on the specific advantages each one of them offers. Such studies not only allow us to understand how biological systems arose and function, but also provide insights into similar processes occurring in other species, sometimes even humans. Hydra, one of the oldest animals to have been used as a model system in biology, is a diploblast, i.e., with a body made up of only two layers of cells, outer ectoderm and inner endoderm, which lives in freshwater bodies. It is a Cnidarian with a simple but defined body plan. It is structurally more complex than sponges, which do not exhibit a defined body axis. Unlike us, who have three major body axes (dorsal-ventral, anterior-posterior, and left-right) and bilateral symmetry, hydra has an oral-aboral axis and radial symmetry. Way back, at the beginning of the eighteenth century, attempts were being made to study marine organisms, such as corals and mollusks, by simulating natural conditions [1]. The breakthrough, however, came, when in the year 1740, Abraham Trembley, who had a $\mathrm{PhD}$ in mathematical infinity, began studying aquatic insects and ended up finding some strange and mobile tubes in water [1]. In the following few years, Trembley studied these strange mobile tubes closely and succeeded not only in keeping these alive in glass jars

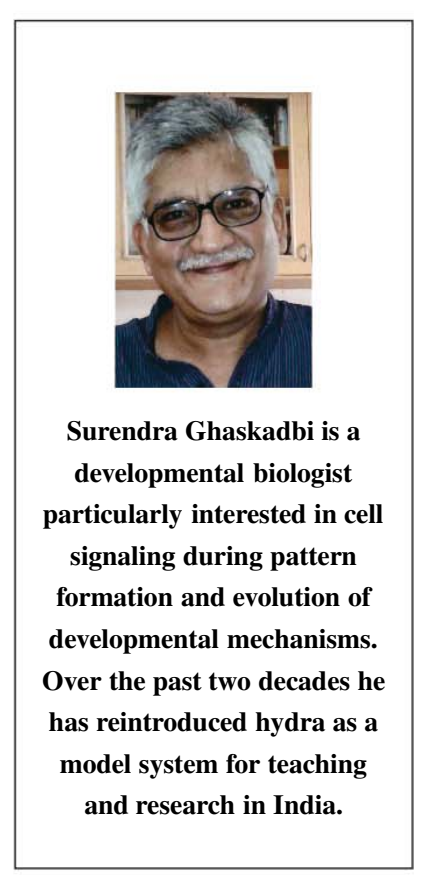

\section{Keywords}

Hydra, regeneration, pattern formation, model system, teaching tool. 
filled with water but also in transporting them over several miles through the postal system. Trembley carried out a large number of experiments with them; these included regeneration of whole polyps from cut pieces and production of the multiheaded hydra. Hydra derives its name from the multiheaded monster in Greek mythology. This is rather unfortunate since there seems to be nothing monstrous about this animal except perhaps to its prey. If one comes across a live hydra, one will immediately appreciate how beautiful, delicate, and lovable it is. Trembley's work, published in 1744, led to what is widely known as the 'Trembley' effect in marine biology [1]. Although hydra itself is a freshwater organism, Trembley's work encouraged scientists to devise strategies to bring, maintain, study, transport, and experiment with marine creatures in the laboratory for the first time.

\section{Morphological and Cellular Features of Hydra}

Hydra is a freshwater diploblastic animal with a simple, yet defined body plan. It has radial symmetry like a starfish and an oral-aboral axis.
As already mentioned, hydra is a freshwater diploblastic animal with a simple, yet defined body plan (Figures 1,2). It has radial symmetry like a starfish and an oral-aboral axis. The oral end has a conical 'hypostome' with a mouth-like opening, surrounded by about 7 tentacles [2]. The number of tentacles varies slightly in individuals of a species and those of different species. Hydra captures its prey with its tentacles, which are richly supplied with the explosive 'nematocytes', the stinging cells-characteristic of Cnidarians (jellyfish, corals, sea anemones, hydra) — that paralyze the prey by injecting poison into them. The aboral end possesses a foot-like structure which has a basal disk; this helps the hydra polyp stick to a surface. Some species of hydra also have a stalklike structure above the basal disk called the 'peduncle'. Between the two ends is the 'body column' enclosing a 'gastric cavity' where food is digested and assimilated. Since there is only one opening to the body; undigested food is egested from the same opening. The lower part of the body column just above the foot is called the 'budding zone' as hydra produces buds in this region during asexual reproduction. Budding is the usual, preferred means of reproduction, although, under unfavorable conditions, 


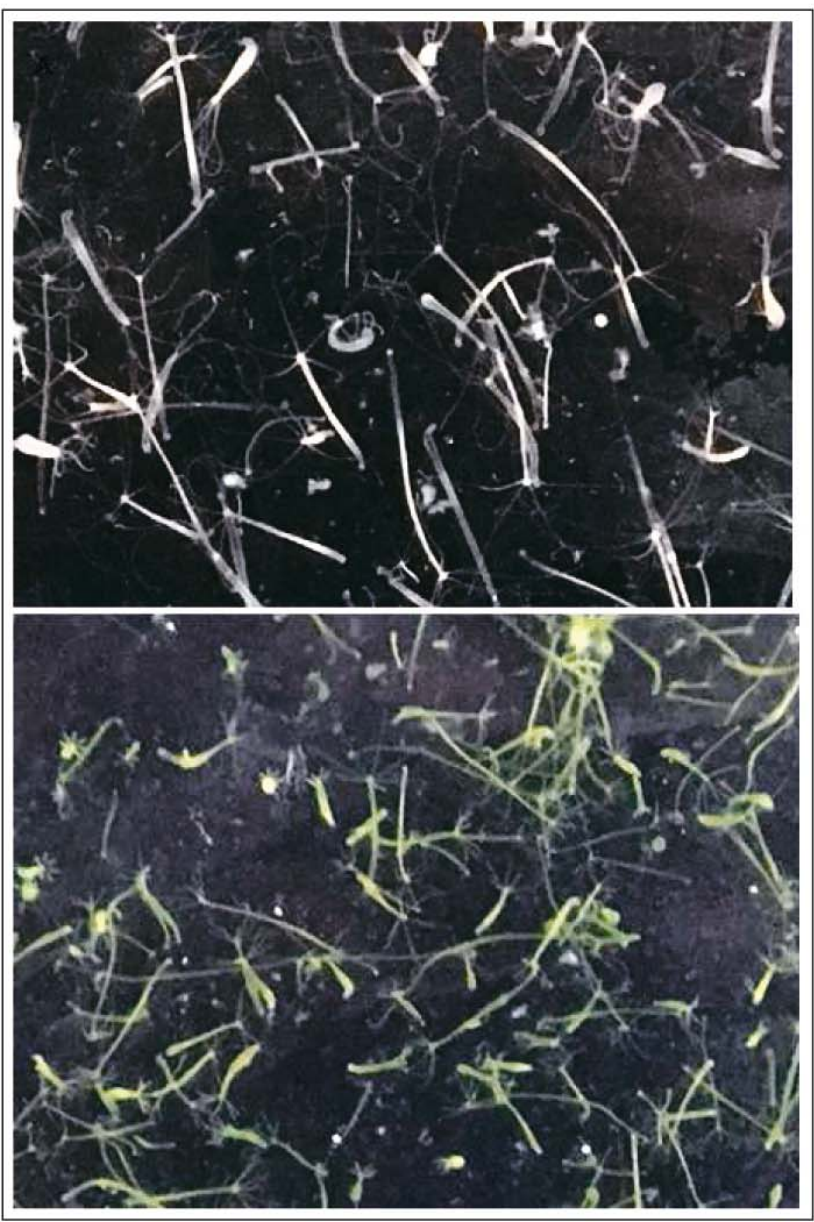

Figure 1. Live hydra polyps in laboratory. (Top) Brown hydra (Hydra vulgaris Ind-Pune) and (Bottom) Green hydra (Hydra viridissima).

such as starvation or cold temperatures, hydra can reproduce sexually. The latter is achieved by producing gametes in newly developed gonads. In some species, both testes and ovary develop in the same polyp, while in some others, they are produced in different polyps. Development begins after the external fertilization of the oocyte by a sperm.

Hydra has 20 to 25 different types of cells (we have about 200 types). Ectoderm and endoderm are mainly made up of ectodermal epithelial and endodermal epithelial cells, respectively. Amongst these are various other types of cells. The nervous system of hydra is in the form of a nerve net made of neurons, spread 


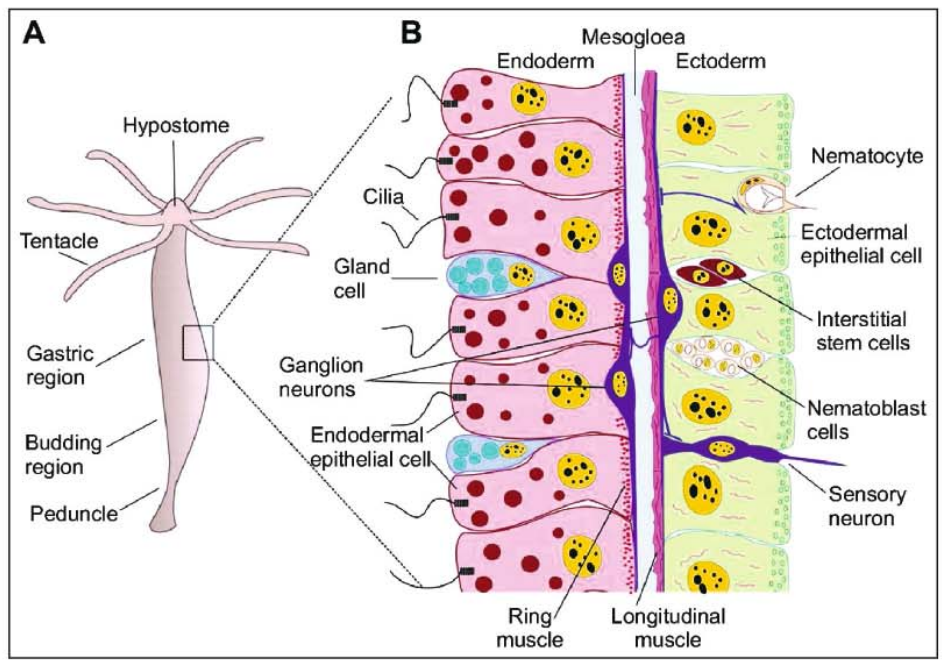

Figure 2. Anatomy of a hydrozoan polyp. (A) A Hydra polyp is a two-layered tube, with a ring of tentacles around the mouth opening at the tip of the hypostome. Asexual budding occurs in the lower half of the body column. Interstitial stem cells and nematoblasts are distributed evenly in the body column, below the tentacle ring and above the border of the peduncle (the stalk between the budding region and the pedal disc). (B) The bilayered cellular organization of the Hydra polyp. Ectoderm and endoderm are separated by an acellular matrix called the mesoglea (gray). All epithelial cells in Hydra are myoepithelial, with myofibers on the basal side (red). In ectodermal epithelial cells (green), the fibers are oriented longitudinally, and in endodermal epithelial cells (pink) they are oriented circumferentially (ring muscle). Most interstitial cells and nematoblast clusters are located between ectodermal epithelial cells. Neurons are found in both the endoderm and ectoderm. Sensory neurons are located between epithelial cells and connect to ganglion neurons (purple), which are at the base of the epithelium on top of the myofibers and sometimes cross the mesoglea. Different types of gland cells, most of which are found in the endoderm, are intermingled with epithelial cells. (Reproduced from U Technau and R E Steele, Evolutionary crossroads in developmental biology: Cnidaria, Development, 138, pp.1447-1458, 2011. doi: 10.1242/dev.048959 with permission from Company of Biologists Limited.)

all over the body. Neurons are more concentrated in the tentacles and around the mouth and help in sensing the prey. The endoderm contains gland cells that help in digestion and mucous cells. 


\section{Hydra as a Perpetual Embryo}

All cells of hydra originate from three distinct stem cell populations. A stem cell is a cell with the ability to self-renew as well as to differentiate into one, two, or more types of progeny (differentiated cells). The three stem cell lineages in hydra are (1) the ectodermal epithelial stem cells, (2) the endodermal epithelial stem cells, and (3) the interstitial or I cells (Figure 3). The epithelial stem cells give rise to the ectodermal and endodermal epithelial cells. They divide every 3 to 4 days. All the other cell types of hydra, the neurons, the nematocytes, the mucous and gland cells, and even the sperm and ova, differentiate from the third stem cell lineage, the I cells, which are dispersed in the ectodermal layer in the middle of the body column and divide much faster (every 1.5 days) than epithelial stem cells. Hydra shows very interesting cellular dynamics. The I cells continuously divide, as they are required to give rise to various kinds of differentiated cells (neurons, nematocytes, mucous cells, gland cells, gametes) [3]. The divided cells begin migrating towards the two extremities (oral and aboral ends) of the body. As they migrate, they differentiate and occupy appropriate positions in the body. All cells are either sloughed off after undergoing apoptosis or programmed cell death in about 20 days from both ends or contribute to the forming bud (Figure 3). This is an important feature of hydra that helps us understand many basic processes during the development of the vertebrate embryo. In vertebrate embryos, cells divide and migrate to occupy different positions within the embryo. They adhere to their neighbors and differentiate to form specialized cells. Many cells undergo programmed cell death in the embryo. These basic cellular processes of cell proliferation, cell migration, cell adhesion, cell differentiation, and cell death often overlap and are essential for normal development. A defect in any of these processes leads to abnormal development. Since the adult hydra polyp exhibits all these cellular processes continuously, it is like a perpetual or everlasting embryo. This peculiarity of hydra allows one to study many embryonic processes in an adult organism. Since hydra is easier to maintain and study in the laboratory than vertebrate em-
Adult hydra polyp is like a perpetual or everlasting embryo. This peculiarity of hydra allows one to study many embryonic processes in an adult organism. 
Figure 3. Cell dynamics in an adult Hydra. Cells divide in the body column, and cell division displaces cells towards the ends of the animal and into buds. Ectodermal and endodermal epithelial cells in the body column give rise to the differentiated ectodermal and endodermal epithelial cells of the tentacles and basal disk. Interstitial stem cells give rise to four classes of differentiated cells. Arrows indicate experimentally determined travelling times of epithelial cells (Campbell 1967b). (Reproduced with the permission of UPV/EHU Press from Martínez and Bridge, Hydra, the everlasting embryo, confronts aging, Int. J. Dev. Biol., 56, pp.479-487, 2012, doi: 10.1387/ijdb.113461dm.)

Grafting a piece of tissue containing several cells from a donor embryo onto a different place in a host embryo allows one to study the effect of the grafted tissue on the host tissue surrounding it.

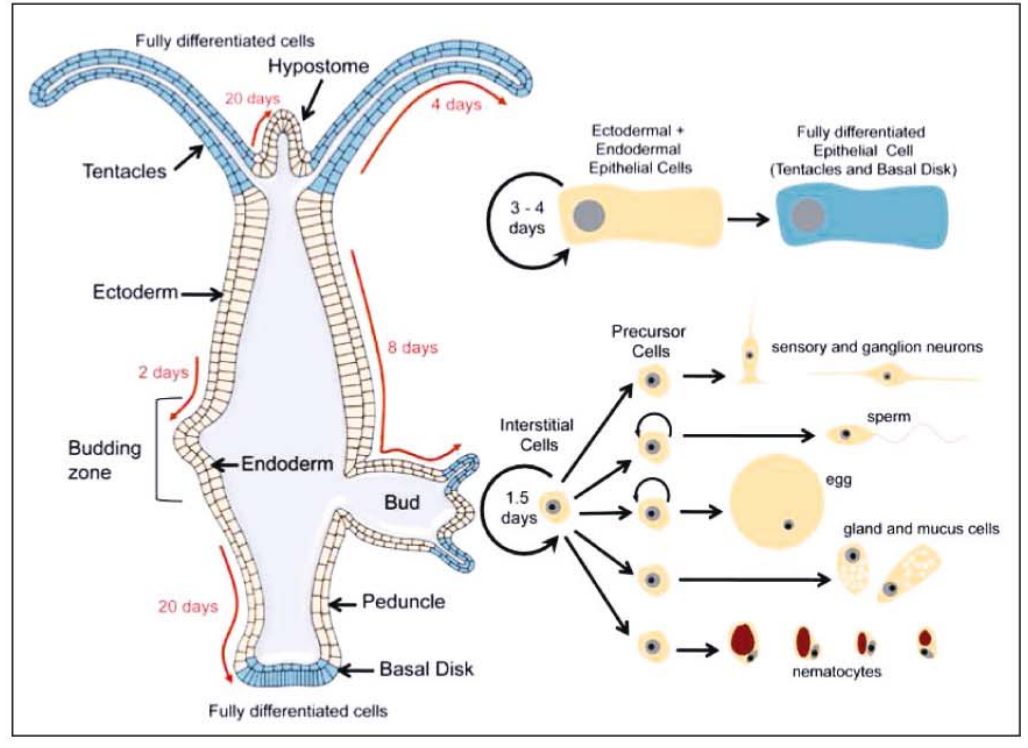

bryos, they serve as a good model in some circumstances.

Very strong evidence for embryo-like characters of the adult hydra polyps is provided by grafting experiments. For over a hundred years, developmental biologists have grafted tissues or cells from one embryo onto or into another to understand how various cell types and tissues originate during development. As the fertilized single-celled zygote undergoes cleavage divisions, more and more cells form. These cells are identical, to begin with, but start becoming different from each other, through a process called cell differentiation (resulting from differential gene expression), since a multicellular organism requires different cell types to perform different functions. Since all the cells of the embryo possess an identical genome, they require detailed instructions to become different from each other, like neurons, skin cells, or liver cells, through the expression of different genes. Instructions required by a cell to become different from other cells, or in other words, to take a particular developmental pathway, originate in the neighboring cells in the embryo. Grafting a piece of tissue containing several cells from a donor embryo onto a different place in a host embryo allows one to study the effect of the grafted tissue on the 
host tissue surrounding it. Arguably, the most famous and spectacular grafting experiment was carried out by Hilde Mangold and Hans Spemann in the early twentieth century. When Mangold grafted a small piece of tissue from the future dorsal side of a donor amphibian embryo onto the future ventral side of a host embryo, it resulted in the formation of a twin embryo. Thus, instructions from the graft cells altered the developmental program of the surrounding host to become dorsal tissue, instead of its normal ventral fate, ultimately forming a new body axis. This process, by which a new embryonic tissue is induced, is known as 'embryonic induction'. The tissue that induces a new axis, in this case, the grafted tissue, is called an 'organizer' (Spemann's organizer in amphibians). Equivalent organizer tissues are identified in other embryos (e.g., Hensen's node in the chick embryo and node in the mammalian embryo). Spemann received the Nobel Prize in Medicine in 1935 for this work. What is interesting to note is that even before Mangold and Spemann's findings, Ethel Browne had carried out similar experiments in hydra, wherein, she transplanted different tissues from a donor hydra onto a host hydra [4]. Her work showed that the hypostome of hydra can induce a new axis in the host. The work has been repeated many times over the years, and we now know that both the hypostome and the foot in hydra possess organizer-like activity (Figure 4 [5]).

\section{Lack of Organismal Senescence in Hydra}

Hydra is peculiar in another way. It does not exhibit organismal senescence, meaning that hydra does not get old and is potentially immortal. People often believe that we age because our cells age. Cells in culture too undergo aging. Defects at the cellular and molecular levels, such as DNA strand breaks, cause aging of cells. Hydra is believed to escape aging because of its three robust stem cell populations, that constantly divide and differentiate, replacing the older cells, thus keeping a hydra polyp forever young. However, some species of hydra do undergo aging after gametogenesis. It may be interesting to note here that so far there has been only one or two reports of hydra developing tumors, and
Hydra does not exhibit organismal senescence, meaning that hydra does not get old and is potentially immortal. 
Figure 4. Contribution of host cells to the secondary axis. H. vulgaris (AEP) expressing GFP either in its ectodermal or endodermal cells were used as hosts and non GFP expressing transplants (a piece of hypostome or a complete foot) were grafted to induce secondary axis. A piece of hypostome $(\mathrm{A}-\mathrm{C})$ and complete foot (D-F) were grafted in the upper, middle and lower parts of the host body column. In all cases, ectodermal and endoderm supports the growth of the secondary axis. (Reproduced from Kadu et al, 2012 with permission.)
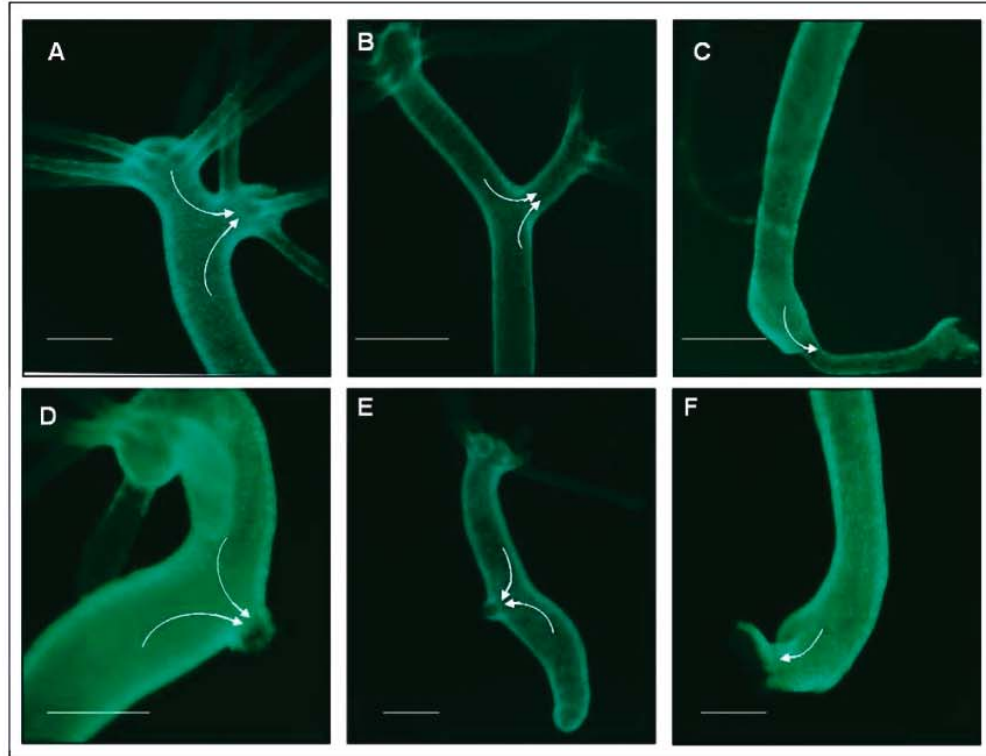

very few natural mutants of hydra exist. In fact, it is also very difficult to experimentally induce stable mutations in hydra. Scientists have now learned to introduce foreign genes into hydra, to produce transgenic strains of hydra with immense potential in hydra biology.

\section{Regeneration in Hydra}

One of the most fascinating features of hydra is its almost unlimited capacity to regenerate the lost body parts. If a hydra polyp is cut into two pieces, the head piece regenerates the lost foot and the foot piece regenerates the lost head.
One of the most fascinating features of hydra is its almost unlimited capacity to regenerate the lost body parts (Figure 5). If a hydra polyp is cut into two pieces, the headpiece regenerates the lost foot and the foot piece regenerates the lost head. This process does not require growth (increase in cell numbers) in hydra, at least in the initial stages; it is, therefore, known as 'morphallaxis', as opposed to epimorphosis, observed in limb and tail regeneration in amphibians, which requires growth. Hence, the resulting regenerate is smaller than the original polyp. Almost all body parts of hydra, except the tentacles and the basal disk, are capable of regeneration to a greater or lesser extent. Even a pellet of hydra cells regenerates into a polyp. Interestingly, if a 
hydra is cut into 3 pieces, the middle piece that lacks both, the head and the foot, regenerates new head and foot precisely on the sides where the original head and foot were present (Figure 5). This means that some information exists in the cells of the middle piece that directs the regeneration of the lost parts in the original orientation. Based on these experiments in hydra, along with limb regeneration experiments in the chick embryo, Lewis Wolpert proposed the theory of morphogen gradients and positional information, in the late nineteen sixties [6]. Morphogens are chemical entities that bring about morphogenesis (development of form and shape). In brief, Wolpert proposed that there exists information in the regenerating middle piece, in the form of concentration gradients of chemicals, that directs the fate of cells towards one (head) or the other (foot) pathway. For example, a gradient of head-forming molecule exists in hydra, wherein the concentration of the molecule is highest at the head end and lowest at the foot end. As a result, in any piece of the body column, the concentration of the head forming molecule will always be highest at the cut end nearer the original head. The same would be true for the foot-forming molecule, but at the opposite orientation. This leads to the regeneration of the lost structures at appropriate sites. Wolpert also proposed that cells present in the gradient can 'sense' their position in a gradient, depending on the concertation of the morphogen they encounter (or are exposed to). Morphogen gradients have since been found to be important and essential for many developmental processes in all organisms. The spectacular regenerative capacity of hydra allows one to study pattern forming mechanisms at morphological, cellular, and molecular levels. Deciphering the mechanisms of regeneration in hydra can provide us with clues regarding the aspects of regeneration that could be useful in understanding why more complex organisms have a significantly diminished capacity to regenerate lost tissues and organs. It is possible that in the future, knowledge of regeneration in hydra may help us find ways to improve the regenerative capacity of other organisms.
Lewis Wolpert proposed the theory of morphogen gradients and positional information, in the late nineteen sixties. Wolpert proposed that there exists information in the regenerating middle piece, in the form of concentration gradients of chemicals, that directs the fate of cells towards one (head) or the other (foot) pathway. 
Figure 5. Regeneration in (A) brown and (B) green hydra. (Picture Courtesy: Saroj Ghaskadbi, Rakhee Vishwakarma, and Alka Chougule.)

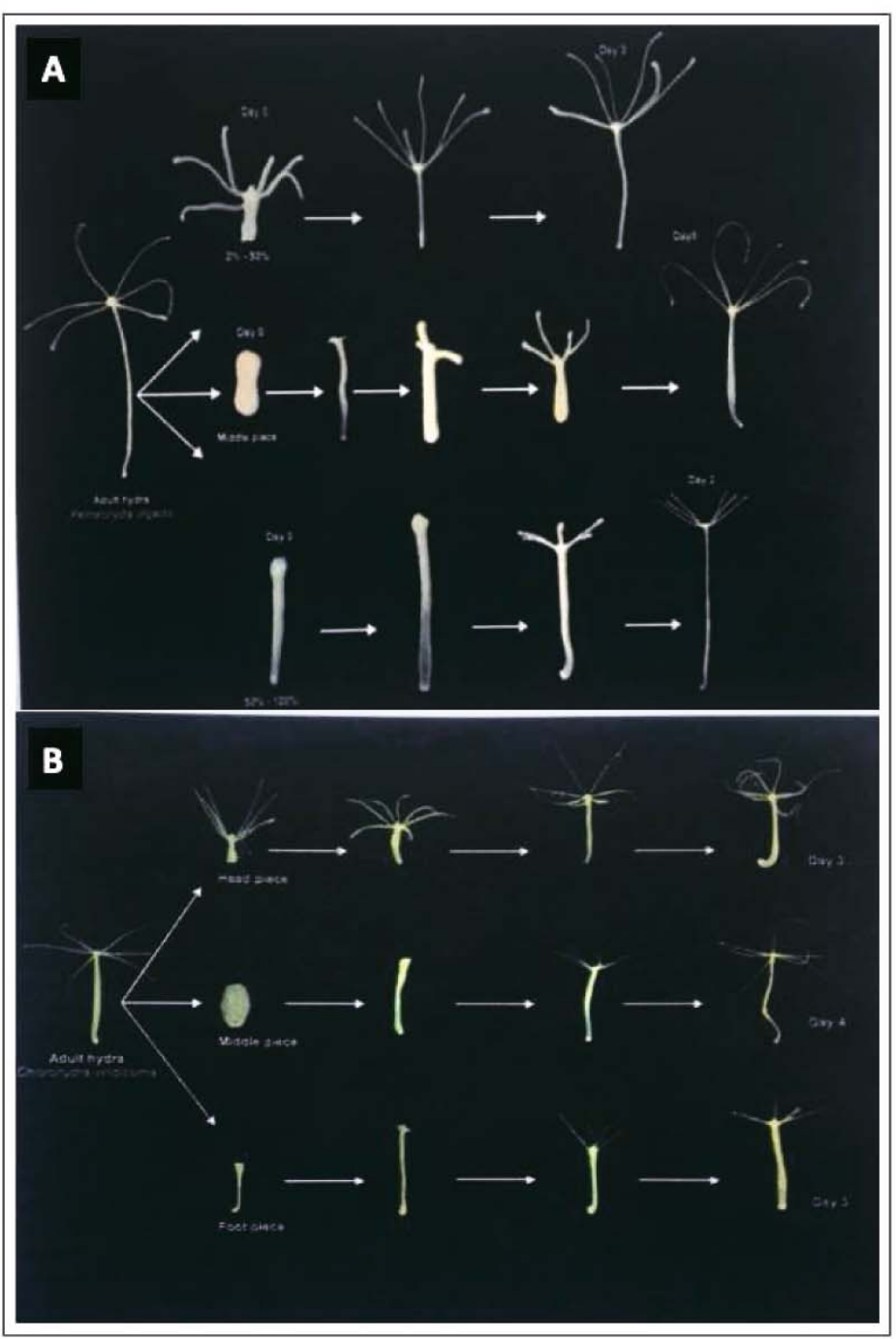

\section{Conservation of Genes and Proteins}

The genome of Hydra magnipapillata was published in 2010. However, much before that, it became evident that many hydra genes and proteins show close structural and sometimes even functional homology with their counterparts from vertebrates, including humans. Let's take a couple of examples to illustrate this point. The first example is cell signaling pathways. In mul- 
ticellular organisms, cells need to talk to or communicate with one another. One way this happens between neighboring cells during early development is through paracrine signaling (signaling between near neighbors). A few signaling pathways, such as TGF- $\beta$, Wnt, Hedgehog, Notch, JAK/STAT, receptor tyrosine kinase [RTK], and nuclear hormone pathways, are believed to be sufficient for embryonic development and pattern formation in animals, including humans. Interestingly, many of these are present in hydra [7]. Hydra is thought to have evolved 60 million years ago (mya), which implies that the signaling pathways are also evolutionarily very old. Although hydra has a relatively simpler body plan than more complex organisms like vertebrates, it still possesses many signaling pathways. In some cases, the hydra genes are also functionally conserved in vertebrates. This is studied by experimentally expressing hydra genes in vertebrate embryos (hetero-specific expression), where they carry out more or less similar functions. Another example is hydra DNA repair genes that code for proteins required to repair the damaged DNA. All organisms have multiple mechanisms to repair damaged DNA since DNA damage can have serious consequences, including cell death, aging, mutations, and cancer. Xeroderma pigmentosum is a genetic disorder in humans in which the ability to repair ultraviolet (UV) radiation-induced DNA damage is severely impaired. Molecules that participate in the repair of UV-induced DNA lesions are Xeroderma pigmentosum A (XPA) through XPG. Interestingly, hydra not only possesses all these genes but also has significant structural homology with their human counterparts. These are just a couple of examples out of several, showing that many of the genes and proteins that evolved in hydra 60 million years ago have been conserved during evolution. Since several genes and proteins that regulate development, regeneration, and pattern formation in hydra have been conserved in almost all animals, including humans, studies in hydra can provide us with clues regarding the evolution of these processes. Hydra is thus a useful model to study the evolution of developmental mechanisms, which is an important and integral part of a new branch of biology called evolutionary developmental biology (evo-devo).
Hydra is thought to have evolved 60 million years ago (mya), which implies that the signaling pathways are also evolutionarily very old. 


\section{Use of Hydra in Teaching}

Hydra can be used to teach many basic biological processes, such as asexual reproduction by budding, and regeneration of tissues. One can design simple feeding, regeneration, and toxicity testing experiments with hydra to excite the curiosity of students, both in colleges and schools.
Hydra can be used to teach many basic biological processes, such as asexual reproduction by budding, and regeneration of tissues. One can design simple feeding, regeneration, and toxicity testing experiments with hydra to excite the curiosity of students, both in colleges and schools. This is particularly true since hydra is easier to collect and maintain in the laboratory than many complex animals. Further, it is also possible to design and implement shortand long-term research projects using hydra in places with limited infrastructure and laboratory facilities. I mention here, a couple of simple approaches to design experiments for pedagogical as well as research purposes as examples. As mentioned, the preferred mode of reproduction in hydra is asexual. Hydra continuously develops buds, which detach once their development is complete. The rate of budding of hydra is proportional to the amount of food they consume. One can design simple experiments in which the rate of budding, and consequently, the doubling of the hydra population, can be studied. Further, during budding, the tentacles of hydra display various intra- and interspecific patterns of emergence which can be studied [8]. This is one of the features often used for taxonomic identification of hydra. Another way of using hydra as a biological model is to study the influence of unnatural culture conditions on hydra. These can be created using varying ambient temperatures or by using exogenous agents. For example, one can introduce exogenous chemicals in the hydra medium and see how this affects the survival, budding, or regeneration in hydra. The concentration of the chemical and duration of exposure can be varied to get an idea of the sensitivity of hydra to altered environments. Because of their high sensitivity to the environment, hydra is a powerful tool to study the toxicity of chemicals and environmental pollutants [9]. Being an aquatic organism, hydra is often a sensitive indicator of pollution in freshwater bodies. A few basic protocols for maintenance of hydra culture and regeneration in hydra are provided at the end of this article.

I would like to add a word of caution, however, based on personal 
experience. Over the past two decades, we have supplied hydra to several colleges and universities in different parts of India. But except for one or two notable exceptions, others have not been able to maintain hydra cultures for any length of time. After the initial enthusiasm, the poor hydra are, sadly, left to starve and disintegrate. One important reason for this is that hydra needs to be fed regularly with live prey and always kept in clean containers. They, in fact, need to be looked after as diligently as one would look after a pet. Teachers and students probably lack the will to do this over long weekends and during vacations. If one wishes to employ this delicate, beautiful and enigmatic animal to inspire students to take up, enjoy and love biology, small sacrifices will have to be made, but in the end, they would certainly be worthwhile.

\section{Protocols}

\section{Hydra Culturing and Maintenance}

Maintain clonal culture of hydra in glass crystallizing dishes containing $1 \mathrm{X}$ hydra medium at a constant temperature of $18 \pm 1^{\circ} \mathrm{C}$ with 12 hour day and night cycle. Feed the polyps with freshly hatched Artemia salina nauplii and change the medium six hours post feeding [10].

\section{Composition of $1 X$ Hydra Medium}

$\mathrm{KCl}-0.1 \mathrm{mM}$

$\mathrm{NaCl}-1.0 \mathrm{mM}$

$\mathrm{MgSO}_{4} .7 \mathrm{H}_{2} \mathrm{O}-0.1 \mathrm{mM}$

$\mathrm{CaCl}_{2} \cdot 2 \mathrm{H}_{2} \mathrm{O}-1.0 \mathrm{mM}$

Tris Base $-1.0 \mathrm{mM}$ (Use $\mathrm{pH} 8.0$ Tris-Cl solution)

\section{Preparation}

Dissolve all the above-mentioned components except $\mathrm{MgSO}_{4}$ (to avoid precipitation) in distilled water. Add separately dissolve
Hydra needs to be fed regularly with live prey and always kept in clean containers. They, in fact, need to be looked after as diligently as one would look after a pet. 
$\mathrm{MgSO}_{4}$ solution to this. Adjust the $\mathrm{pH}$ to 8.0. Then make up the final volume to $1 \mathrm{~L}$ and autoclave for 40 minutes. It would be convenient to prepare a stock of 100X hydra medium and dilute with distilled water to $1 \mathrm{X}$ for use.

\section{Artemia Nauplii Hatching}

Soak Artemia cysts in saltwater in a beaker (3.2 $\mathrm{g}$ of rock salt dissolved in $1 \mathrm{~L}$ of tap water) under continuous aeration. Depending on ambient temperature, cysts will hatch within 24 to $48 \mathrm{~h}$. Since these crustaceans are attracted to strong light, i.e., photophilic, freshly hatched larvae can be easily collected from the beaker by exposing it to light from one side.

Before feeding, wash Artemia well with tap water to remove excess salts. Feed approximately 20 Artemia per hydra. After 6 h, clean the bowl by changing the hydra medium.

Tissue Manipulations (Hydra bisection, trisection, and regeneration)

Place $24 \mathrm{~h}$ starved hydra on a clean glass slide for relaxing

$$
\downarrow
$$

Cut hydra with a sharp blade or needle into two/three pieces

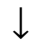

Keep all these pieces (head, middle and base pieces) separately in a six well plate containing hydra medium

Observe after $24 \mathrm{~h}$ for head and foot regeneration

Head pieces will regenerate a foot, middle pieces will regenerate both head and foot, and the foot pieces regenerate a head. Head regeneration can be easily observed with the appearance of tentacle rudiments. Foot regeneration can be observed by the presence of a sticky secretion through which it starts adhering to the substratum. Foot-specific staining can be done after $48-72 \mathrm{~h}$ of regenerating head and middle pieces to observe functional foot formation. 


\section{Foot-specific Staining}

Mucous cells in the basal disk of hydra contain a peroxidase-like enzyme that allows specific staining of these cells using substrates for peroxidases. Here, we use ABTS as a substrate for peroxidase. This enzyme provides an excellent marker for mucous cell differentiation in the regenerating foot [11].

\section{ABTS Solution}

Citric Acid $-65.5 \mathrm{mM}$

Tri-sodium citrate $-34.5 \mathrm{mM}$

ABTS (2,2'-azino-bis (3-ethylbenzothiazoline-6-sulphonic acid)) $-0.1 \%$

Hydrogen peroxide $-0.003 \%$.

After $48-72 \mathrm{~h}$, foot regenerating pieces can be stained to monitor the formation of a functional foot.

\section{Protocol for Foot-specific Staining}

Take head pieces that are regenerating foot, in a six well plate, about 10 pieces per well.

Remove hydra medium completely and add $2-3 \mathrm{ml}$ of ABTS solution containing hydrogen peroxide.

$\downarrow$

Cover the plates with aluminum foil and incubate for 5-15 min at RT till the pink coloration develops

$$
\downarrow
$$

Stop the reaction by adding $1 \mathrm{X}$ PBS (pH 5.0) or wash them with distilled water.

$$
\downarrow
$$

After 15-20 min wash, transfer these head pieces to fresh PBS and observe. A purple colored ring can be seen at the regenerating foot tip.

Note: Since 2018, MACS-Agharkar Research Institute has made available a hydra culture kit for the first time in India. The kit
Mucous cells in the basal disk of hydra contain a peroxidase-like enzyme that allows specific staining of these cells using substrates for peroxidases.

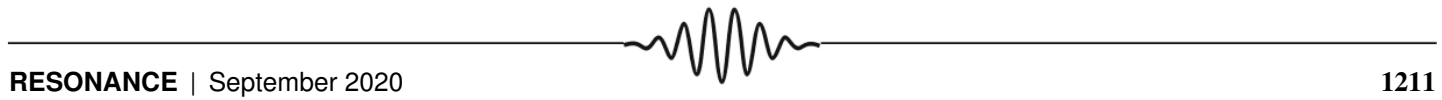


consists of hydra polyps, 10X hydra medium, Artemia cysts, rock salt, crystallizing bowls and Pasteur pipettes. Supply of the kit, temporarily suspended due to the COVID-19 pandemic, will be resumed as soon as possible. For cost and other details, please visit: http://aripune.org/.

\section{Acknowledgements}

I am grateful to Vidya Patwardhan and Satyajit Rath for critical reading of the draft manuscript and suggesting changes. I thank Dr Sujata Deshpande, Ms Rohini Londhe, and Mr Mahadeo Daware for various inputs. Over the past two decades, my hydra laboratory has been funded by the Departments of Science \& Technology (DST) and Biotechnology (DBT), Science and Engineering Research Board (SERB), Government of India, and MACS-Agharkar Research Institute. Surendra Ghaskadbi is an Emeritus Scientist of Council for Scientific and Industrial Research, New Delhi

\section{Suggested Reading}

[1] M J Ratcliff, The Trembley effect or the birth of marine zoology, Int, J, Dev. Biol., 56, pp.425-436, 2012.

[2] U Technau and R E Steele, Evolutionary crossroads in developmental biology, Development, 138, pp.1447-1458, 2011.

[3] D E Martínez and D Bridge, Hydra, the everlasting embryo confronts aging, Int, J, Dev. Biol., 56, pp.479-487, 2011.

[4] E N Browne, The production of new hydranths in hydra by the insertion of small grafts, J. Exp. Zool, 7, pp.1-23, 1909.

[5] V Kadu, S S Ghaskadbi, and S Ghaskadbi, Induction of secondary axis in hydra revisited, Int. J. Mol. Cell. Med., 1, pp.11-20, 2012.

[6] N Vargesson, Positional information - A concept underpinning our understanding of developmental biology, Dev. Dyn., DOI10.1002/dvdy.116.

[7] S Ghaskadbi, Cell signaling molecules in hydra: insights into evolutionarily ancient functions of signaling pathways, Int. J. Dev. Biol., 64, pp.141-149, 2020.

[8] R Londhe, L S Krishnapati, and S Ghaskadbi, Description and phylogenetic characterization of hydra from Naukuchiatal (Uttarakhand, India) and comparison with other hydra strains, Curr. Sci., 113, pp.1739-1745, 2017.

[9] V Patwardhan and S Ghaskadbi, Invertebrate alternatives for toxicity testing: Hydra stakes its claim, ALTEX Proc., 2, pp.69-76, 2013. 
[10] T Sugiyama and T Fujisava, 1977, Genetic analysis of developmental mechanisms in hydra I. Sexual reproduction of Hydra magnipapillata and isolation of mutants, Dev. Growth Diff., 19, pp. 187-200, 1977.

[11] S Hoffmeister and H Chica Schaller, A new biochemical marker for footspecific cell differentiation in hydra, Roux's Dev Biol., 194, pp.453-461, 1985.
Address for Correspondence Surendra Ghaskadbi Developmental Biology Group MACS-Agharkar Research Institute

G.G. Agarkar Road Pune-411 004 India.

Email: ghaskadbi@gmail.com 